\title{
Traduire
}

Une autre perspective sur r tr traduction

Revue française de la traduction

$221 \mid 2009$

Voies de l'interprétation

\section{Le Prix Pierre-François Caillé de la Traduction}

\section{Florence Herbulot}

\section{OpenEdition}

\section{Journals}

Édition électronique

URL : http://journals.openedition.org/traduire/358

DOI : 10.4000/traduire.358

ISSN : 2272-9992

\section{Éditeur}

Société française des traducteurs

\section{Édition imprimée}

Date de publication : 15 décembre 2009

Pagination : 104-111

ISSN : 0395-773X

\section{Référence électronique}

Florence Herbulot, «Le Prix Pierre-François Caillé de la Traduction », Traduire [En ligne], 221 | 2009, mis en ligne le 12 novembre 2013, consulté le 26 novembre 2020. URL : http://journals.openedition.org/ traduire/358 ; DOI : https://doi.org/10.4000/traduire.358 


\section{Le Prix Pierre-François Caillé de la Traduction}

\section{Florence Herbulot}

Pour la plupart d'entre nous, Pierre-François Caillé (1907 - 1979) n'est, au mieux, qu'un nom : un personnage flou, si lointain, et tellement vieux...

II est vrai que le 23 septembre 2009 était le trentième anniversaire de sa disparition : trente ans, une bonne génération et le pouce.

Mais pour un certain nombre, pour ceux qui, jeunes diplômés arrivant sur le marché ou praticiens confirmés, ont eu l'occasion et la chance de travailler avec lui, il demeure bien vivant, bien présent, un exemple remarquable et l'artisan de ce qu'est aujourd'hui le métier de traducteur. Vous me direz qu'il reste beaucoup à faire, c'est vrai, mais maintenant c'est votre tour d'œuvrer.

En 1980, lorsque le comité de la SFT entreprit de créer un prix de traduction, il avait en vue plusieurs objectifs.

II s'agissait d'abord, évidemment, de perpétuer la mémoire de l'homme récemment disparu, de celui qui avait, dès 1947, créé, "inventé " la traduction en tant que profession, avec un petit groupe disparate de traducteurs, littéraires pour la plupart, ou d'ingénieurs venus à la traduction technique, et qui avait su en quelques années faire de cette association confidentielle un organisme reconnu. II avait également réussi à mettre en place une organisation internationale, la Fédération Internationale des Traducteurs, créée en 1953, reconnue par l'UNESCO, et partie prenante dans la rédaction et l'adoption par cet organisme de la Recommandation de Nairobi, premier texte où le traducteur se voit accorder le statut d'auteur.

Il avait, pour cela, mis en œuvre toute son énergie, son art de la diplomatie, ses relations, sa force de persuasion. Et il avait mené ses créations à un niveau dont ses prédécesseurs n'auraient pas osé rêver, un niveau dont nous bénéficions tous depuis lors.

Ce traducteur littéraire et de cinéma - on le connaît, en particulier, pour la traduction du roman Autant en emporte le vent, et du film qui en fut tiré, mais il est l'auteur du doublage de plus de 300 films - avait été président de la SFT pendant 23 ans, et de la FIT, à plusieurs reprises et 
jusqu'à sa disparition. On comprendra donc aisément que nous nous soyons sentis orphelins après son départ.

Un autre objectif de la création de ce prix était de donner du relief au métier de traducteur, souvent mal perçu : pour beaucoup d'utilisateurs de la traduction, dans le domaine littéraire comme dans le domaine technique, celui qui la réalise était, et reste encore, bien souvent, considéré comme annexe, accessoire, remplaçable. Pour nous, traducteurs professionnels, c'était assez dur à supporter !

Nous voulions que l'importance de notre rôle soit mieux reconnue, et pas uniquement en littérature où, à la limite, notre nom figure (presque toujours...), ce qui nous assure un peu de notoriété. C'est pourquoi nous avons voulu que le lauréat du Prix puisse être un traducteur non littéraire, d'où notre principe d'alternance entre les années "fiction " et " non-fiction ".

Désolée, nous n'avons pas trouvé mieux que "non-fiction ", mais nous sommes ouverts à toute suggestion.

Enfin, comme il existait déjà quelques prix de traduction, mais destinés à couronner une carrière méritoire, nous souhaitions attribuer notre Prix à un traducteur débutant, jeune ou n'ayant pas atteint la notoriété.

On voit d'emblée que ces deux objectifs d'ordre technique sont difficiles à atteindre. En effet, l'édition de livres techniques ou scientifiques est nettement moins prolifique que l'édition de romans, et quant à la condition de début de carrière, il est relativement rare qu'un éditeur fasse confiance à un débutant pour la traduction d'ouvrages intéressants.

Tout cela complique beaucoup la tâche de notre jury, qui doit jongler avec ces différents éléments.

Mais parlons un peu du jury. II a été présidé jusqu'en 1983 par l'adorable Agnès Doniol, puis cette fonction m'est échue en 1984. Ses membres sont choisis par cooptation, et se renouvellent parfois, pour des raisons professionnelles ou personnelles : par exemple, Muriel Valenta, ayant été admise comme traductrice à l'ONU, à New York, a dû nous quitter cette année. Ce sont pour l'essentiel des traducteurs, des interprètes, des universitaires ; le président de la SFT en fait partie ès qualités, et demeure parfois des nôtres après la fin de son mandat. Tous sont dévoués à la tâche, qu'ils assument avec courage et assiduité, mettant beaucoup de sérieux dans le jugement de la qualité des livres qu'ils ont à lire chaque année.

Pour sa composition, nous avons d'abord fait appel à des amis de Pierre-François Caillé et, au fil des ans, à des personnalités diverses, mais toujours intéressées par la traduction : écrivains comme Régine Deforges, François Billetdoux, Jean Blot, Alain Guillemou, Michèle Kahn, 
Claudine Jardin, Vénus Khoury-Ghata, Jean Rousselot, Paul Fournel, Jacques Cellard ; universitaires comme Sylvère Monod, Raymond Las Vergnas, Maurice Gravier, Danica Seleskovitch ; scientifiques, parfois académiciens, comme Pierre Lépine, André Caquot, Bernard Frank, Pierre Agron... Je ne peux pas les citer tous, mais nous avons toujours cherché à rassembler des jurés capables d'évaluer les ouvrages techniques ou scientifiques aussi bien que purement littéraires. C'est une règle que nous continuons d'appliquer pour la recherche de nouveaux jurés.

Nous voulons aussi avoir parmi nos membres des personnes connaissant ou pratiquant des langues très diverses, pour pouvoir recourir éventuellement à la version originale, afin de résoudre les mystères que pourrait présenter la traduction. Mais pour des professionnels, la qualité de la traduction est en général évidente. II faudrait que le traducteur se soit donné beaucoup de mal pour divaguer au point qu'on ne puisse plus s'apercevoir de ses fantaisies ! Toutefois, le risque existe, et nous avons donc la possibilité de demander à l'éditeur communication de l'ouvrage initial, ce que nous faisons parfois.

Pour la sélection des livres, la procédure est la suivante : nous écrivons à une liste d'éditeurs, mise à jour chaque année, en leur demandant de nous communiquer les ouvrages répondant à nos critères. Dès réception de leur réponse, et après vérification des caractéristiques du traducteur, nous leur demandons deux exemplaires qui sont soumis à des membres du jury pour une lecture rapide. Cette étape franchie, le jury se réunit pour établir la liste finale des ouvrages en lice, qui doivent donc satisfaire à nos différents critères : traduction de bonne qualité, traducteur en début de carrière, parution dans les deux ans précédents. Ce choix est parfois difficile, comme en 2004 (année " fiction ") où nous avons écarté onze livres sur les dix-sept ouvrages reçus.

Nous demandons ensuite un nombre d'exemplaires suffisant pour diffusion auprès de l'ensemble du jury, qui va pouvoir lire à tête reposée les 4,5 ou 6 livres retenus, parmi lesquels il désignera le lauréat de l'année au cours d'une réunion finale.

Bien sûr, rien n'interdit à un traducteur de nous signaler un ouvrage qu'il juge digne de concourir, qu'il en soit ou non l'auteur : à nous alors de prendre contact avec l'éditeur pour réaliser l'opération. À bon entendeur...

Un coup d'œil à la liste des lauréats depuis 1981 montre avec quel éclectisme le jury travaille. Même si nous y trouvons - c'est inévitable, surtout pour les ouvrages techniques - bon nombre de traductions de l'anglais, des langues plus rares y figurent aussi : russe, japonais, turc tchaghatay, grec, tchèque, roumain, vieil islandais, et puis espagnol et italien, bien sûr.

La lecture de cette même liste fait apparaître quelques vides, sur lesquels on peut s'interroger. Par exemple, il n'y a pas de lauréat entre 1994 et 2000. C'est que notre prix a connu, de 
1995 à 1998, une période de dormance, puis la SFT m'a demandé de le relancer. Nous avons redonné vie au jury, cherché et trouvé un certain nombre de personnes pour remplacer celles qui ne pouvaient plus nous aider, et le Prix a de nouveau été décerné en septembre 2000.

Autre vide, l'année 2001, suivie d'un double prix en 2002 : les livres proposés cette année-là pour la catégorie "non-fiction " étant trop peu nombreux, et de qualité trop décevante, le jury a décidé de reporter à 2002 l'attribution du prix, en même temps que celle du prix " fiction ". C'est ainsi que furent couronnées deux traductrices, l'une du russe (Nathalie Amargier, Le Pingouin, roman) et l'autre du roumain (Odile Serre, Composition aux parallèles inégales, essai).

À sa création, le montant du prix était de 5000 F. Nous avons bénéficié, jusqu'en 1993, d'une subvention de l'Académie française. De 1982 à 1994, l'association avec la Société des Gens de Lettres nous a permis de recevoir une contribution annuelle, d'où le doublement de la dotation, passée ainsi de 5000 à 10000 F. Nous bénéficions toujours du concours de l'ESIT. Après le passage à l'euro, la SFT a porté le prix à 2000 euros.

Malgré toute la gentillesse des éditeurs, il est rare que le livre primé reçoive la bande qui donnerait, en librairie, un relief à notre prix. Ce fut le cas, en 1983, pour La Cellule insolite (Daniel Allibert-Kouraguine, trad. de l'anglais, Interéditions), et en 2006 pour La Petite Renarde rusée (Michel Chasteau, trad. du tchèque, Éd. Fayard). II faut dire que la difficulté est grande : les livres sont déjà distribués, on ne peut les récupérer pour poser la bande ; pour que ce soit possible, il faut ou il faudrait une réédition, mais comment l'obtenir?

Nos lauréats n'ont pas tous fait carrière dans la traduction; certains, surtout en matière de livres techniques ou scientifiques, sont des spécialistes qui poursuivent leur chemin dans le domaine qui est le leur. D'autres sont devenus écrivains, comme Roselyne Majesté-Larrouy (Récit des temps perdus, trad. du grec, Éd. du Seuil, 1984) ou Myrian Chirousse (Le Roi transparent, trad. de l'espagnol, Éd. Métailié, 2008).

Certains ouvrages nous ont beaucoup appris, comme Le Palais de mémoire de Matteo Ricci (Martine Leroy-Battistelli, trad. de l'anglais, Éd. Payot, 1987), sur les phénomènes et les moyens mnémotechniques, ou Mesurer le Monde - L'incroyable histoire de l'invention du mètre (Martine Devillers-Argouac'h, trad. de l'anglais, Éd. Flammarion, 2005), riche de révélations sur le monde scientifique dans les années de la Révolution.

Celui qui recevra le prix 2009, Le Secret de la Grande Pyramide (Julien Le Bonheur, trad. de l'anglais, Éd. Fayard) devrait aussi passionner tous ceux que l'Égypte attire et fascine. Quant à son traducteur, qui est tout à fait " en début de carrière ", nous lui souhaitons un brillant avenir dans ce métier où il nous rejoint. 
Maintenant, en avant pour le Prix 2010 ! II couronnera la traduction d'un roman, paru depuis 2008, et peut-être aurez-vous, lecteurs, une part dans son choix, en nous communiquant les références d'ouvrages qui vous semblent bien traduits - ou que vous avez traduits vousmêmes. À nous, ensuite, de vérifier que nos critères de sélection soient satisfaits...

\section{Le Prix de la Traduction Pierre-François Caillé}

Prix fondé en 1981 par la Société Française des Traducteurs.

Décerné avec le concours de

l'École Supérieure d'Interprètes et de Traducteurs (ESIT)

de l'Université Paris III - Sorbonne Nouvelle

\section{Règlement du Prix}

Ce prix, fondé par la Société Française des Traducteurs à la mémoire de Pierre-François Caillé, Président d'honneur de la SFT dont il fut l'un des créateurs et président fondateur de la Fédération Internationale des Traducteurs, est destiné à récompenser, alternativement, une traduction d'une œuvre littéraire ou d'un ouvrage scientifique, technique ou de vulgarisation, parue au cours des vingt-quatre mois précédents.

Ce prix annuel d'un montant de DEUX MILLE EUROS a une double vocation : récompenser un traducteur en début de carrière ou n'ayant pas encore atteint la notoriété, et attirer l'attention sur la profession de traducteur, métier qui exige un travail consciencieux, des connaissances diverses et approfondies, et qui mérite d'être reconnu.

Le président du jury devra être un membre actif de la SFT, élu pour deux ans à la majorité relative avec mandat renouvelable, sur proposition du Comité directeur de la SFT.

En cas de vacance d'un siège, le jury désignera un nouveau titulaire sur proposition du Comité directeur de la SFT.

Aucun membre du jury ne pourra recevoir le prix. Aucun membre du Comité directeur de la SFT ne pourra recevoir le prix.

Pour le choix du lauréat, le vote par délégation est admis. En cas de non-accord, la voix du président est prépondérante.

Le prix sera décerné chaque année à l'occasion de la Journée mondiale de la traduction.

En cas de non-attribution, le jury pourra attribuer deux prix l'année suivante.

Société Française des Traducteurs

22 rue de la Pépinière - 75008 PARIS

0142939996 [secretariat@sft.fr] 


\section{Liste des lauréats}

1981

Jean-Louis Bacqué-Grammont

Le Livre de Babur

Turc tchaghatay

Presses Orientalistes de France

1982

Amina Okada

Tristesse et Beauté

Japonais

Albin Michel

1983

Daniel Alibert-Kouraguine Anglais

La Cellule insolite

Interéditions

1984 Roselyne Majesté-Larrouy Grec

Récit des temps perdus

Seuil

1985

Odile Demange

Anglais

Lucy

Robert Laffont

1986

Mario Carelli

Chronique de la Maison assassinée

Portugais

A.M. Métailié/Mazarine

1987 Martine Leroy-Battistelli Anglais

Le Palais de mémoire de Matteo Ricci

Payot

Bomarzo

Séguier

1989

Catherine Ballestero

Espagnol

La Révolution des Saints

Belin

Anglais

Une Saison à Hakkâri

Gallimard

1991 François-Xavier Dillmann

Vieil islandais

L'Edda de Snorri Sturluson

Gallimard

1992 Anne-Marie Tatsis-Botton

Russe

Carnets d'un toqué

L'Âge d'Homme

Ana Gerschenfeld

Anglais

Biologie de la conscience

Odile Jacob 


$\begin{array}{ll}1994 & \begin{array}{l}\text { Daniel Struve } \\ \text { Japonais }\end{array} \\ 2000 & \begin{array}{l}\text { Nathalie Amargier } \\ \text { Russe }\end{array} \\ 2002 & \begin{array}{l}\text { Odile Serre } \\ \text { Roumain }\end{array} \\ & \text { Diane Weill Ménard } \\ & \text { Italien } \\ & \\ 2003 & \text { Julie Sauvage } \\ & \text { Italien } \\ 2004 \quad & \text { Marc Weinstein } \\ & \text { Russe } \\ 2005 & \text { Martine Devillers-Argouac'h } \\ & \text { Anglais }\end{array}$

2006 Michel Chasteau

Tchèque

2007 Justine Landau

Anglais

2008 Myriam Chirousse

Espagnol

2009 Julien Le Bonheur

Anglais
Le vent se lève

L'Arpenteur

Le Pingouin

Liana Levi

Composition aux parallèles inégales

Maurice Nadeau

Lamarck, Genèse et enjeux du transformisme, 1770-1830

CNRS Editions

Lima, Pékin, Venise... 1688, une année dans le monde

Autrement

La Prise d'Izmail

Fayard

Mesurer le Monde - L'incroyable histoire de l'invention du mètre

Flammarion

La Petite Renarde rusée

Fayard

Echolalies - Essai sur l'oubli des langues

Seuil

Le Roi transparent

Métailié

Le Secret de la Grande Pyramide

Fayard 


\section{Liste des membres du Jury 2009}

Présidente : Mme Florence HERBULOT, traductrice, Maître de conférences honoraire à l'Université Paris III.

Et, par ordre alphabétique :

M. Pierre AGRON, ancien président du Comité d'étude des termes techniques français.

Mme Suzanne BOIZARD, traductrice.

Mme Huguette BRUSICK, traductrice.

Mme Béatrice COMMENGÉ, traductrice littéraire.

Mme Chris DURBAN, traductrice.

Professeur Fortunato ISRAËL, traducteur.

Mme Maria LEBRET-SANCHEZ, traductrice.

Professeur Marianne LEDERER, interprète de conférence.

M. Philip MINNS, interprète de conférence.

Professeur Jean-Luc MOREAU, écrivain, poète, traducteur.

Mme Héloïse NEEFS, lexicographe.

M. Gilles OUVRARD, traducteur, interprète de conférence.

Mme Caroline SUBRA - ITSUTSUJl, traductrice, Présidente de la Société Française des Traducteurs. Mme Marianne VÉRON, traductrice littéraire.

M. Nicolas VÉRON, traducteur, conseiller à l'Assemblée nationale, chargé de cours à l'INALCO. 